\title{
Adesão a um programa de atenção à saúde bucal para crianças e adolescentes infectados pelo HIV e atitude dos responsáveis
}

\author{
Adherence to an oral health program for HIV infected children \\ and adolescents and the attitudes of their caretakers
}

Fernanda Campos M achado ${ }^{1}$

I vete Pomarico Ribeiro de Souza ${ }^{1}$

Luiz Fernando Rangel Tura ${ }^{2}$

Glória Fernanda Castro $^{1}$

${ }^{1}$ Departamento de Odontopediatriae Ortodontia, Faculdadede Odontologia, Universidade Federal do Rio deJaneiro. Av. Brigadeiro Trompowisky s/n, Cidade Universitária, Ilha do Fundão. 21941-590 Rio de Janeiro RJ.

fercampo@terra.com.br ${ }^{2}$ Departamento deM edicina Preventiva, Faculdade de M edicina, Universidade Federal do Rio deJaneiro.
Abstract Thisstudy aimed to evaluate theadherence to an Oral Health Program (OHP) for HIV infected children and adolescents, as well as the attitudes of their caretakers regarding oral care. A total of 58 caretakers that accompany the children in medical appointments at an AIDS ambulatory were interviewed for collecting personal data and data regarding adherence to the $\mathrm{OH}$ P or other odontological treatment and attitudes related to oral care. Approximately $70 \%$ of the caretakers stated that their children participated in the OHAP, however $20 \%$ of them did not return to the recall appointments; such visits were even less frequent when the caretakers were not the parents themselves $(p=0.036)$. The adherence of this population to dental treatment outside the OHP was small, $48 \%$ of the caretakers stated that the child did not conclude the treatment when referred to another place for treatment. The attitude of the caretakers regarding dental care of HIV + children was not considered satisfactory. Furthermore, it is very important to have pediatric dentists in the multi-professional teams that attend HIV + children and adolescents and to promotethis program among the parents and all medical teams involved with such patients.

Key words HIV, Oral health, Patient participation, Attitude towards health, Children
Resumo 0 estudo avaliou a adesão a um Programa deAtenção à SaúdeBucal (PASB) por crianças e adolescentes infectados pelo HIV, bem como a atitude de seus responsáveis quanto a cuidados em relação à saúde bucal. Foram entrevistados 58 responsáveis que acompanhavam crianças e adolescentes HIV + nas consultas médicas de rotina no ambulatório de aids, os quais foram indagados sobre os dados pessoais, adesão ao PASB ou a outros serviços odontológicos e atitudes quanto aos cuidados bucais. A proximadamente $70 \%$ dos responsáveis disseram quea criança participava do PASB; entretanto, 20\% delas não retornavam às consultas de prevenção quando solicitadas, e este retorno era menor quando os responsáveis não eram os próprios pais $(p=0,036)$. A adesão desta população ao tratamento odontológico fora do PASB foi pequena, visto que $48 \%$ dos responsáveis relataram que a criança não concluiu o tratamento quando este não foi realizado no PASB. A atitude dos responsáveis em relação aoscuidados bucais dascrianças HIV + também não foi considerada satisfatória. D esta forma, ressalta-se a importância da inserção do odontopediatra em equipes multidisciplinaresqueatendem criançaseadolescentes HIV +, bem como da divulgação deste serviço para os familiares e para todas as equipes médicas envolvidas com estes pacientes.

Palavras-chave HIV, Saúde bucal, Participação do paciente, Atitude frente à saúde, Criança 
Introdução

A epidemia da ai ds no Brasil, apesar de estar em processo de estabilização, encontra-se ainda em patamares elevados, tendo atingido, até junho de 2006, um total de aproximadamente $16 \mathrm{mil}$ crianças infectadas no país, de acordo com os dados do último boletim epidemiológico ${ }^{1}$.

A experiência decáriee gengivite em crianças infectadas pelo HIV é alta, sendo causada tanto por problemas inerentes à doença, quanto por fal ta deinformações em relação a práticas deatenção à saúde bucal, que levam a hábitos inadequados de higiene ${ }^{2}$. Além disso, por na maioria das vezes apresentarem um nível socioeconômico e cultural baixo, 0 acesso destes pacientes a cuidados de saúde bucal é deficiente ${ }^{3,4}$.

O controle da saúde bucal éum componente importante no manejo de pacientes com infecção pelo HIV. A ausência de uma cavidade bucal saudável pode afetar a qualidade de vida, complicar o tratamento das condições médicas e criar ou exacerbar problemas nutricionais e psicossociais $s^{5}$

Para minimizar estas complicações, foi criado um Programa de Atenção à Saúde Bucal (PASB) no ambulatório de aids do serviço de pediatria de um hospital universitário do Rio de Janeiro. 0 atendimento odontológico é realizado no próprio ambulatório médico, no dia da consulta médica mensal da criança. Este atendimento visa orientar os responsáveis e os pacientes em relação a cuidados sobre saúde bucal, bem como realizar escovação supervisionada e aplicação tópica de flúor. A cada trimestre, é realizado exame bucal, sendo feito tratamento restaurador atraumático (ART) se necessário. Quando o paciente necessita de outros procedimentos odontológicos que não são realizados no PASB, como exodontias e restaurações, este é encaminhado para outros serviços odontológicos.

Quando sealmeja o restabelecimento ea pre servação da saúde bucal destas crianças, a ade são a estes programas de atenção a saúde bucal, bem como a colaboração de todo núcleo familiar, são de grande importância. Embora vários modelos em relação à adesão tenham sido aplicados em investigações de saúde geral e adesão a regimes de promoção de saúde na medicina, como por exemplo adesão à terapia antiretroviral ${ }^{6-8}$, pouco se sabe sobre adesão a recomendações etratamentos em saúde oral entre pacientes infectados pelo HIV. Além disso, muitas vezes a ati tude ea prática não condizem com 0 aprendizado ou com os ensinamentos, que podem não ser adequados ${ }^{8}$. Desta forma, o presente estudo tem como objetivo avaliar a adesão a este programa de atenção à saúde bucal por crianças e adolescentes infectados pelo HIV, bem como a atitude dos responsáveis pelos mesmos quanto a cuidados em relação à saúde bucal.

\section{Métodos}

Este estudo teve como base o modelo epidemiológico observacional do tipo seccional, sendo utilizado como instrumento de coleta de dados um roteiro de entrevista individual estruturado. Foram entrevistados 58 responsáveis, selecionados por conveniência em um período de dois meses, que acompanhavam crianças e adolescentes infectados pelo HIV nas consultas médicas de rotina no ambulatório de aids do serviço de pediatria do hospital universitário da Universidade Federal do Rio de Janeiro (UFRJ). Este ambulatório é um centro de referência para 0 atendimento de crianças e adolescentes infectados pelo HIV e conta com aproximadamente 340 pacientes em tratamento. 0 estudo foi realizado com 0 objetivo de avaliar o programa de atenção a saúde bucal já existente no local, observando as ne cessidades de introdução de outras formas de controle das doenças bucais, com o intuito de aprimorar este programa, levando as sugestões e problemas a programas similares existentes. Este estudo foi aprovado pelo Comitê de Ética em Pesquisa do IPPM G da UFRJ e todos os responsáveis assinaram o termo de consentimento livree esclarecido.

0 instrumento de pesquisa foi um formulário com perguntas abertas e fechadas contendo informações referentes aos dados pessoais, adesão ao Programa de Atenção à Saúde Bucal (PASB) ea outros serviços odontológicos eatitudes quanto a cuidados bucais, aplicado a cada responsável sob a forma de entrevista, realizada por um único pesquisador (Quadro 1).

Esteinstrumento foi testado previamenteem um grupo de dez responsáveis por pacientes da clínica de odontopediatria da mesma universidade, visando adequar o formulário e treinar a pesquisadora. A pós o pré-teste, seis questões foram alteradas e três foram acrescentadas ao formulário.

Todas as respostas das questões abertas foram reorganizadas em categorias de análise ${ }^{9}$, sendo posteriormente inseridas e analisadas, juntamente com as respostas das questões fechadas no programa Epi-Info 3.3.2 (versão para Win- 
dows). Foi realizada uma análise descritiva dos dados e foram utilizados testes (teste qui-quadrado; teste exato de Fisher) para as associações entre as variáveis, com significância de $5 \%$.

\section{Resultados}

Dos 58 responsáveis entrevistados (idade média 37,5 anose desvio-padrão 12,2) , 65,5\% (38) eram os próprios pais das crianças. Deste total, $81 \%$ (47) apresentavam baixo nível de escolaridade (analfabeto a ensino fundamental completo) e o número médio de filhos na família era de 2,8 e desvio-padrão 1,7 crianças, com a distribuição de média de idade de 7,8 anos e desvio-padrão 3,1.
Quando os responsáveis foram questionados se conheciam o Programa de Atenção Saúde Bucal do ambulatório de aids do hospital universitário, a maioria respondeu positivamente (72,4\%; 42). Em relação à participação no PASB, $70,7 \%$ (41) disseram que a criança participava; dentre estes, $80,5 \%$ (33) relataram saber o que era realizado, descrevendo os procedimentos conforme citado na Tabela 1 . Somente um responsável relatou que a criança participava de outro programa preventivo que não o mencionado neste estudo.

Quando questionados se algum dentista já havia orientado a criança quanto aos cuidados necessários para se evitar a cárie, 81\% (47) dos responsáveis responderam positivamente e foi

Quadro 1. Formulário utilizado para a entrevista com os responsáveis pelas crianças infectadas pelo HIV.

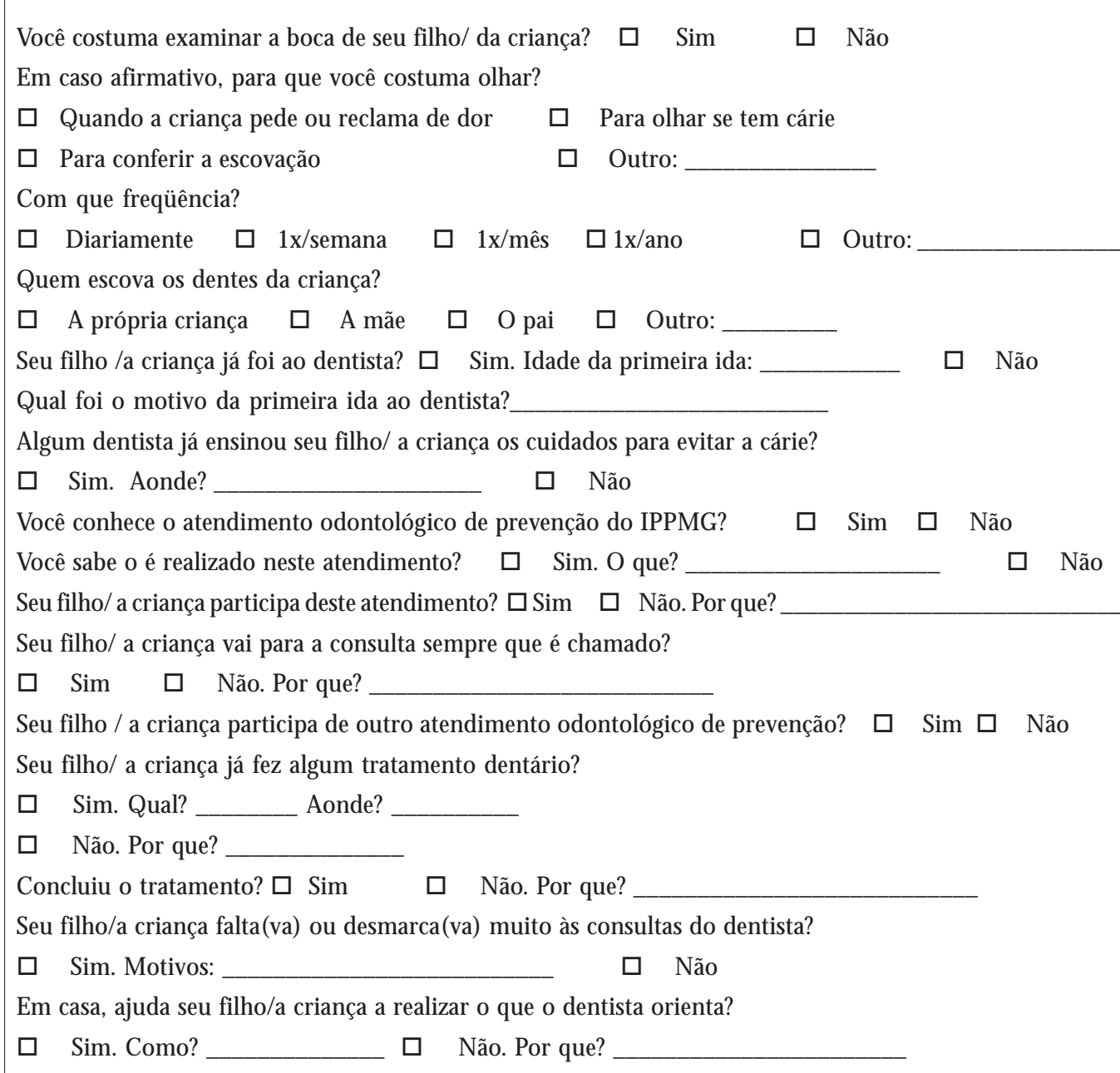


encontrada relação com a participação do menor no Programa de Atenção à Saúde Bucal $(p=0,0099)$ (Gráfico 1).

D os responsáveis que relataram ter suas crianças participantes do PASB ( $n=41), 80,5 \%$ (33) informaram que elas retornavam às consultas deprevenção quando solicitadas; entretanto, este retorno foi menor quando os responsáveis não eram os próprios pais $(p=0,0036)$ (Gráfico 2$)$.

A maioria $(91,4 \%)$ dos entrevistados relatou que a criança já fora ao dentista, sendo que a primeira visita ocorrera na faixa etária de 1 a 9 anos, média de 4,5 anos e desvio-padrão 2,5 . 0 s principais motivos relatados para a primeira visita foram: presença de cárie/dor (45,2\%), encaminhamento médico $(26,4 \%)$ e prevenção $(15,1 \%)$.

Tabela 1. Procedimentos realizados no Programa de Atenção à Saúde Bucal descritos pelos responsáveis $(n=33)$.

\begin{tabular}{lc}
\hline \multicolumn{1}{c}{ Procedimento relatado } & Freqüência \\
Escovação & $66,6 \%(22)$ \\
Exame & $57,6 \%(19)$ \\
Aplicação de flúor & $54,5 \%(18)$ \\
ART & $9,1 \%(3)$ \\
Orientações & $6,1 \%(2)$ \\
\hline
\end{tabular}

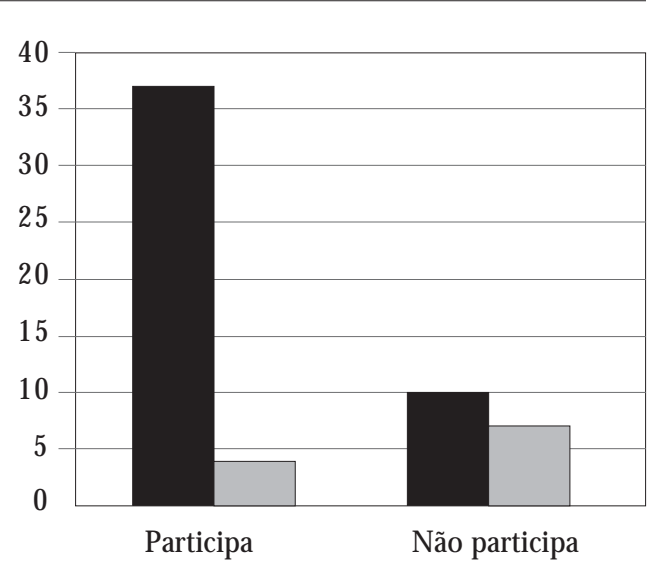

Recebeu orientação $\mathrm{p}=0,0099$; Teste $\square$ Não recebeu orientação

Exato de Fisher

Gráfico 1. Relação entre relato de recebimento de orientação profissional prévia e participação no Programa de Atenção à Saúde Bucal.
Dentre os responsáveis cujas crianças já foram submetidas a algum tratamento odontológico $(63,2 \%$ / 36), os procedimentos relatados incluíram, principalmente, restaurações (63,9\%) e exodontias $(58,3 \%)$ e, em quase todas as vezes $(83,3 \%)$, este tratamento foi realizado em uma instituição pública. Segundo 48,6\% (17) destes entrevistados ( $n=36)$, as crianças não terminaram o tratamento odontológico iniciado fora do PASB, devido, principalmente, a dificuldades financeiras $(29,4 \%)$ e à presença da doença aids $(17, \%)$.

Das crianças quenunca realizaram tratamento $(n=22)$, os principais motivos relatados para este fato foram: não necessidade (60\%) e dificuldades financeiras (15\%). Os outros motivos incluíram problemas desaúde dos responsáveis ou da criança, dificuldade de acesso aos serviços públicos e falta de tempo.

A maior parte dos entrevistados $(89,7 \%)$ relatou que examinava a boca da criança, sendo que, dos que examinavam, $69,2 \%$ eram os próprios pais e $30,8 \%$, outros responsáveis. 0 principal motivo deste exame (Tabela 2) foi conferir a escovação (73,1\%), mas somente $46,2 \%$ (24) dosqueexaminavam o faziam diariamente. Apesar de 70,7\% (41) dos responsáveis relatarem que auxiliavam as crianças no cuidado preventivo caseiro, na maioria das vezes ( $86,2 \% ; 50$ ), a criança realizava sozinha a escovação.

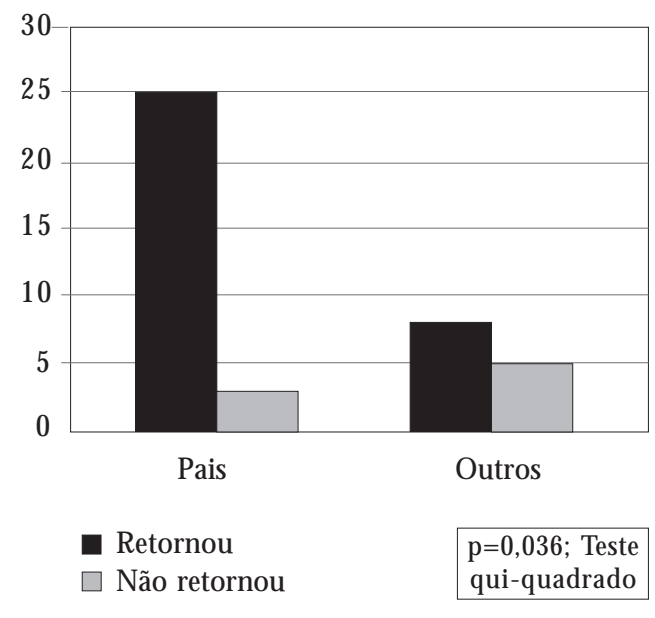

Gráfico 2. Relação entre retorno ao Programa de Atenção à Saúde Bucal e tipo de responsável presente na consulta médica. 
Tabela 2. M otivos que levavam os responsáveis a examinar a boca da criança $(n=52)$.

\begin{tabular}{ll}
\hline \multicolumn{1}{c}{ Motivo relatado } & Freqüência \\
\hline Para conferir escovação & $73,1 \%(38)$ \\
Para verificar a presença de cárie & $28,8 \%$ (15) \\
$\begin{array}{l}\text { ou outra alteração } \\
\text { Quando a criança pede ou reclama } \\
\text { dedor }\end{array}$ & $21,2 \%$ (11) \\
\hline
\end{tabular}

\section{Discussão}

Estudos sobre a saúde bucal de pacientes de grupos socioeconômico e cultural menos favorecidos indicam que estes apresentam um deficiente acesso a cuidados odontológicos quando comparados com pacientes de níveis mais elevados ${ }^{3,6}$ 10,11. Crianças e adolescentes infectados pelo HIV $(\mathrm{HIV+)}$ geralmente pertencem a estes grupos desfavorecidos ${ }^{3,4}$ e isto foi observado no presente estudo, no qual $81 \%$ dos responsáveis entrevistados apresentavam baixo nível de escolaridade.

É importante que serviços de atenção à saúde bucal, como é o caso do Programa de Atenção a Saúde Bucal (PASB) descrito neste artigo, sejam desenvolvidos para esta população ${ }^{10}$. Sendo assim, a adesão das crianças e adolescentes HIV + a estes programas, assim como o envolvimento de todo núcleo familiar, é essencial para a melhoria do status de saúde bucal destes pacientes. Os resultados do presente estudo indicam que 70,7\% da amostra de crianças e adolescentes H IV + selecionados participavam do PASB oferecido, o que foi considerado uma boa participação, embora aproximadamente $20 \%$ destas não retornem às consultas de prevenção quando solicitadas.

Observou-se também que a adesão desta população ao tratamento odontológico fora do PASB foi menor, visto que $48,6 \%$ dos responsáveis relataram que a criança não concluiu o tratamento quando este foi iniciado em outros serviços, corroborando com os achados de Broder et al. ${ }^{3}$, os quais verificaram queo grau de colaboração entregrupo decrianças HIV + ébaixo, apresentando elevadas taxas de abstenção e abandono ao tratamento. Tal fato pode ser explicado pela presença de uma doença potencialmenteletal, o que leva muitas famílias a negligenciarem os cuidados de manuten ção de saúde bucal, privilegiando a desordem sistêmica principal ${ }^{12,}{ }^{13}$. Fatos como estes servem para ressaltar a impor- tância de programas de atenção à saúde bucal dentro do mesmo ambulatório, onde crianças e adolescentes fazem o tratamento médico, e da inserção deste serviço na equipe multidisciplinar que atende a pacientes infectados pelo HIV.

A literatura médica relata que a aderência ao tratamento anti-retroviral é reconhecida como um problema clínico de grande relevância nos pacientes HIV + e está diretamente ligada à vida familiar da criança HIV $+8,14$, sendo que esta geralmente apresenta uma falta de suporte familiar, fazen do parte de famílias desestruturadas por falecimento, abandono ou doenças de seus pais. Dentro destecontexto, nesteestudo, pôde-seperceber quea adesão ao PASB foi significantemente maior quando os responsáveis eram os próprios pais das crianças.

A importância da existência do PASB para estas crianças pôde ser demonstrada pela relação significativa encontrada entre o recebimento de orientação sobre saúde bucal ea participação no PASB (Gráfico 1), sugerindo que a maioria das crianças participantes recebeu esta orientação em função do programa oferecido no ambulatório. Entretanto, apesar da orientação fazer parte do PASB, al guns responsáveis não tinham a percepção de estar sendo orientados, o que leva a crer que tal orientação não tem sido bem entendida, necessitando reformulações.

0 conhecimento sobre o que é realizado no PASB pelos responsáveis não foi satisfatório. Embora a maioria deles $(80,5 \%)$ tenha relatado saber o que é realizado, eles não conseguiram descrever o que de fato é executado (Tabela 1). Este resultado serviu para alertar a equipe responsável pelo PASB sobre a necessidade de oferecer maior atenção aos responsáveis, orientando-os individualmente em relação ao funcionamento do programa com o objetivo de conseguir uma maior integração da família e conseqüente adesão da criança.

A atitude dos responsáveis em relação aos cuidados bucais das crianças eadolescentes HIV + também foi avaliada e os resultados não foram muito satisfatórios, pois apesar de $88,7 \%$ dos entrevistados relatarem que examinam a boca de suas crianças com o principal objetivo deconferir a escovação, somente $46,2 \%$ destes fazem esta conferência diariamente e a maioria das crianças $(86,2 \%)$ realiza a escovação sozinha. Esta deficiência na atenção caseira pode refletir na condição bucal destas crianças, acarretando elevada prevalência decárie, conforme demonstrado por outros estudos ${ }^{2,15}$. Isto corrobora o fato de que 0 principal motivo relatado para a primeira visita 
ao dentista foi a presença de cárie edor e os principais procedimentos odontológicos realizados nestas crianças foram exodontias e restaurações.

Conclui-se, a partir dos resultados encontrados, que a adesão ao PASB foi boa, embora pudesse ter alcançado um maior comprometimento dos participantes. No entanto, no caso de tratamentos odontológicos realizados fora do programa, essa adesão foi ainda menor. Além disso, pode-se concluir que as atitudes dos responsáveis por estas crianças em relação à saúde bucal não foi adequada, necessitando um maior estímulo e envolvimento dos mesmos.

Neste contexto, torna-se imprescindível a conscientização dos responsáveis por estas crianças da importância da saúde bucal para uma maior e melhor sobrevida destes pacientes, bem como fazêlos entender que são participantes ativos para a melhora da saúde bucal e qualidade de vida de suas crianças.

Também deve ser ressaltada a importância da participação do odontopediatra em equipes multidisciplinares que atendem a crianças eadolescentes infectados pelo HIV, bem como a todos os pacientes com comprometimento sistêmico, proporcionando-Ihes um atendimento integral. Da mesma forma, deve ser destacada a importância da divulgação deste serviço para toda a equipe médica envolvida com estes pacientes. Assim, almeja-se uma adesão mais satisfatória das crianças e adolescentes infectados pelo HIV a estes programas, bem como uma maior colaboração dos responsáveis para a manutenção da saúde bucal destes pacientes. 


\section{Colaboradores}

FC M achado, na qualidade de autora principal, desenvolveu a metodologia, sendo responsável pela aplicação do instrumento de coleta de dados, análise, interpretação e discussão dos mesmos, bem como pela redação do artigo científico; LFR Tura trabalhou na elaboração do instrumento, análise, interpretação e discussão dos dados e revisão crítica do artigo; IPR de Souza e GFR de Castro, na qualidade de orientadoras, participaram do delineamento metodológico, da discussão e análise dos dados e da revisão final da versão a ser publicada.

\section{Referências}

1. Brasil. M inistério da Saúde. 2006. Programa nacional de DST e AIDS. [acessado 2007 Fev 07]. Disponível em: http://www.aids.gov.br

2. Ribeiro AA, Portela M, Souza IP. Relação entre biofilme, atividade de cárie e gengivite em crianças HIV +. Pesqui Odontol Bras 2002; 16(2):44-50.

3. Broder HL, Catalanotto FA, Reisine $\mathrm{S}$, Variagiannis E. Compliance is poor among HIV infected children with unmet dental needs. Pediatr Dent 1996; 18(2):137-138.

4. Brown JB, Rosenstein D, Mullooly J, Rosetti MO Robinson S, Chiodo G. Impact of intensified dental care on outcomes in human immunodeficiency virus infection. Aids Patient Care and DSTs 2002; 16(10): 479-486.

5. AIDS Institute. Oral Health Care for Adults, Adolescents and Children with HIV infection. $1^{\text {st }}$ ed. Albany, N.Y: New York State Department of Health; 1998.

6. Broder HL, Russel EV, Reisine ST. Oral health perceptions and adherence with dental treatment referral among caregivers of children with HIV. AIDS Education and Perceptions 1999; 11(6):541-551.

7. Ellis DA, Naar-King S, Cunningham PB, Secord E. Use of multisystemic therapy to improve antiretroviral adherence and health outcomes in HIV-infected pediatric patients: evaluation of a pilot program. AIDS Patient Care and DSTs 2006; 20(2):112-121.

8. Nicholson O, Mellins C, Dolezal C, Brackis-Cott E, Abrams EJ. HIV treatment-related knowledge and selfefficacy among caregivers of HIV-infected children. Patient Education and Counseling 2006; 61:405-410.

9. Minayo MCS. O desafio do conhecimento: pesquisa qualitativa em saúde. 8 a ed. São Paulo: Hucitec; Rio de Janeiro: Abrasco; 2004.

10. Bolden AJ. Implications of access, utilization and need of oral health care by low income groups and minorities on the dental delivery system. Journal of Dental Education 1993; 57(12):888-98.

11. Ismail AI, Sohn W. The impact of universal dental care on disparities in caries experience in children. JADA 2001; 132:295-303.

12. Roberts IF, Roberts GJ. Dental disease in chronically sick children. J Dent Child 1981; 48(5):346-351.

13. Castro GF, Castro RAL, Souza IP. Paciente pediátrico com doença sistêmica: considerações importantes do atendimento primário ao paciente especial. Revista do Centro de Estudos FO/UERJ 2001; $7(2): 42-46$.

14. Brackis-Cott E, M ellins CA, Abrams E, Reval T, Dolezal C. Pediatric HIV medication adherence: the views of medical providers from two primary care programs. Journal of Pediatric H ealth Care, 2003; 17(5):252-260.

15. Hicks MJ, Flaitz CM, Carter AB, Cron SC, Rossmann SN, Simon CL, Demmler CJ, Kline M W. Dental caries in HIV-infected children: a longitudinal study. Pediatr Dent 2000; 22(5):359-364.

Artigo apresentado em 07/08/2006

Aprovado em 17/11/2006

Versão final apresentada em 03/07/2007 